\title{
EL CONTROL INTERNO Y LA INFORMACIÓN FINANCIERA DE UNA ENTIDAD DEL ESTADO: MINISTERIO DE TRABAJO Y PROMOCIÓN DEL EMPLEO
}

\author{
INTERNAL CONTROL AND FINANCIAL INFORMATION OF A STATE ENTITY: \\ MINISTERIO DE TRABAJO Y PROMOCIÓN DEL EMPLEO
}

Melissa Jennifer Puma Hancco Universidad Nacional Mayor de San Marcos Lima, Perú

ORCID: http://orcid.org/0000-0002-8910-5954 Correo electrónico: needmeli68@hotmail.com

\section{RESUMEN}

Objetivo: Comprobar los componentes de la Ley 28716 que influyen en la fiabilidad de la información financiera del Ministerio de Trabajo y Promoción del Empleo, periodo 2014-2016. Método: Es una investigación de tipo deductiva, no experimental-transversal-correlacional. Resultados: Los resultados de la encuesta al personal que labora en el Ministerio de Trabajo y Promoción del Empleo, arrojan que un adecuado Sistema de Control Interno bajo los componentes de Ambiente de Control mejorará positivamente la consistencia de la información que sustenta el activo, pasivo y patrimonio del Estado de Situación Financiera. Conclusiones: La Evaluación de Riesgos mejorará la coherencia y confiabilidad de la información que sustenta los ingresos y gastos el Estado de Gestión; las actividades de control mejorará positivamente la oportunidad del movimiento patrimonial de la información que sustenta el Estado de Cambios en el Patrimonio Neto; la información y comunicación mejorará positivamente la razonabilidad de la información del movimiento del efectivo que sustenta el Estado de Flujo de Efectivo y el monitoreo mejorará que la información esté libre de errores sustanciales de las notas aclaratorias a los Estados Financieros.

Palabras clave: Control; información; finanzas; supervisión; rendición.

\begin{abstract}
Objective: To determine to what extent the lack of internal control under the components of "Law 28716" influences the reliability of the financial information of the Ministry of Labor and Employment Promotion, period 2014-2016. Method: It is a deductive, not experimental-transversal-correlational investigation. Results: The results of the survey of the personnel working in the Ministry of Labor and Employment Promotion, show that an adequate Internal Control System under the Control Environment components will positively improve the consistency of the information that sustains the active, passive and equity of the Statement of Financial Position. Conclusions: The Risk Assessment will improve the coherence and reliability of the information that sustains the income and expenses of the State of Management; the control activities will positively improve the opportunity of the patrimonial movement of the information that sustains the State of Changes in the Net Worth; the information and communication will positively improve the reasonableness of the cash movement information that sustains the Cash Flow Statement and the monitoring will improve that the information is free of substantial errors of the explanatory notes that support the Notes to the Financial Statements.
\end{abstract}

Keywords: Control; information; finances; supervision; surrender.

( - Los autores. Este artículo es publicado por la Revista Quipukamayoc, Universidad Nacional Mayor de San Marcos. Este es un artículo de acceso abierto, distribuido bajo los términos de la Licencia Creative Commons Atribución-NoComercial-Compartirlgual 4.0 Internacional.(http://creativecommons.org/licenses/by-nc-sa/4.0/), que permite el uso no comercial, distribución y reproducción en cualquier medio, siempre que la obra original sea debidamente citadas. 


\section{INTRODUCCIÓN}

El Ministerio de Trabajo y Promoción del Empleo se crea el 30 de abril de 1949 mediante Decreto Ley N 11009; es un organismo del poder ejecutivo con personería jurídica de derecho público y responde a sus principales áreas programáticas de acción relacionadas con la defensa de los derechos fundamentales en materia socio laboral, salud y seguridad en el trabajo, así como la promoción del empleo y autoempleo, inspección del trabajo, reconversión e intervención laboral, formación y capacitación profesional, certificación y normalización de competencias laborales, mercado de trabajo e información laboral, concertación laboral, diálogo social y seguridad social.

El Comité de Organizaciones patrocinadoras de la Comisión de Caminos (COSO, 2013) refiere que:

El control interno es un proceso llevado a cabo por el consejo de administración, la dirección y el resto del personal de una entidad, diseñado con el objeto de proporcionar un grado de seguridad razonable en cuanto a la consecución de objetivos relacionados con las operaciones, la información y el cumplimiento (p. 1).

Siguiendo los parámetros de los componentes del Sistema de Control establecido en la Ley de Control Interno de las entidades del Estado (LEY N. ${ }^{\circ}$ 28716), en el 2014, se realizó un examen a los estados financieros por la firma Sandoval Aliaga y Asociados (SOA), en ese contexto la administración es la responsable de establecer y mantener una estructura de control interno. En ese sentido, toda estructura de control interno persigue como objetivo, entre otros, proporcionar a la administración con razonable, pero no absoluta seguridad que las transacciones se ejecuten de acuerdo con autorizaciones de la administración y se encuentren enmarcadas en las disposiciones legales establecidas.

Como resultado al examen sobre los estados financieros, se detectó ciertas deficiencias que constituyen una oportunidad de mejora de control. Así se tiene que:

- Respecto de las rendiciones de fondo para pagos en efectivo del ejercicio 2014, se han realizado pagos no establecidos en la directiva interna vigente.

- Incumplimiento de lo establecido en las normas vigentes, respecto a las rendiciones y depósitos de los saldos no utilizados por concepto de viáticos de la revisión realizada al análisis de los saldos de la subcuenta 1205.0501 viáticos, del rubro 1205 servicios y otros pagados por anticipado al 31 de diciembre de 2014 .
- Existencia de Errores en las Provisiones de Contingencias Judiciales.

Estos problemas conllevan a preguntarnos $\underset{i}{ }$ Un adecuado ambiente de control interno asegura que la información que sustenta el activo, pasivo y patrimonio del Estado de Situación Financiera del Ministerio de Trabajo y Promoción del Empleo sea consistente?

Para ello se revisó la siguiente teoría: Según la Contraloría General de la República (2014), el control interno es un proceso integral efectuado por el titular, funcionario y servidores de una entidad, diseñado para enfrentar los riesgos y para dar seguridad razonable de que, en la consecución de la misión de la entidad, se alcanzaran los objetivos de la misma, es decir, es la gestión misma orientada a minimizar los riesgos.

Por otro lado, Gamboa, Puente y Ysidora (2016) manifiestan que el control interno ha sido reconocido como una herramienta para que la dirección de todo tipo de organización obtenga una seguridad razonable para el cumplimiento de sus objetivos institucionales y esté en capacidad de informar sobre su gestión a las personas interesadas en ella.

El control interno se puede entender como un programa de organización desarrollado a partir de un conjunto de métodos, procesos y procedimientos que adopta y coordina una empresa con el fin de salvaguardar sus bienes, realizar una comprobación eficaz de sus datos contables con el objetivo de determinar su grado de confianza a efectos de desarrollar y consolidar una administración eficiente y lograr el cumplimiento de los objetivos organizacionales.

Mendoza, García, Delgado y Barreiro (2018) afirman que:

El control interno busca promover la eficiencia, eficacia, transparencia y economía en las operaciones de la entidad, la calidad de los servicios públicos que presta, cuidar y resguardar los recursos y bienes del Estado contra cualquier forma de pérdida, deterioro, uso indebido y actos ilegales, en general, contra todo hecho irregular o situación perjudicial que pudiera afectarlos (p. 212).

El control interno es un sistema integral que posee un conjunto de procesos integrados, mas no es un conjunto de mecanismos burocráticos que efectúa la alta dirección, la administración y el personal de toda organización. Entonces, el control interno garantiza la consecución de los objetivos organizacionales.

En ese contexto, se puede intuir que la aplicación del control interno en cualquier órgano del Gobierno Central, regional, local o cualquier entidad pública resulta trascendente debido a su importancia en el establecimiento 
de controles para encontrar, priorizar y contrarrestar los riesgos asociados a las actividades organizacionales.

Es por ello, que el control interno es un plan asociado a un conjunto de métodos, procesos y procedimientos que tienen como fin salvaguardar sus bienes, verificar la exactitud y seguridad de los datos de contabilidad, desarrollar la eficiencia de las operaciones y fomentar el apego a la política administrativa prescrita.

En el artículo 3 de la Ley de Control Interno de las entidades del Estado LEY No 28716 se define al sistema de control interno de la siguiente manera: Conjunto de acciones, actividades, planes, políticas, normas, registros, organización, procedimientos y métodos, incluyendo la actitud de las autoridades y el personal, organizados e instituidos en cada entidad del Estado, para la consecución de los objetivos indicados en el artículo 4 de la presente Ley.

En ese contexto, el término control, en su acepción más extendida, hace referencia a comprobación, inspección, fiscalización o intervención, es decir, "consiste en contrastar si unas actuaciones están o no en consonancia con normas o criterios dados que marcan cómo se debe hacer; todos estos conceptos son aplicables a la gestión de la actividad económica-financiera" (Gutiérrez y Católico, 2015, p. 556).

Asimismo, según el Instituto Americano de Contadores Públicos Certificados (AICPA) el control interno es el proceso efectuado por la junta de directores, la gerencia y el personal de una entidad, diseñado para proporcionar seguridad razonable respecto al logro de objetivos (Fonseca, 2011).

Es más factible y sencillo encontrar posibles errores que detectar fraudes, puesto que los fraudes son cometidos a través de actos que se conciben para ocultar su existencia. En ese sentido, el control interno puede ayudar a las entidades a detectar errores, fraudes y demás riesgos que afecten la continuidad de las actividades de la entidad.

A nivel público y privado, el Control Interno Marco usado es el Integrado a COSO, ya que permite a las organizaciones el desarrollo de forma eficiente y efectiva de la aplicación de un sistema de control interno adaptado a los cambios del entorno operativo y de negocio.

Según Dextre y Del Pozo (2012), el control interno debe cubrir los siguientes aspectos:

a. Implementación de normas y aspectos para la descripción y ejecución de las actividades, con los controles pertinentes que las aseguren, así como las diversas formas de medir el rendimiento. b. Comprobación de las actividades y determinar si los resultados guardan concordancia con los objetivos inicialmente establecidos.

c. Medición y evaluación de los resultados con respecto a las metas que se alcanzaron durante determinado periodo.

d. Ejecución de acciones correctivas de ser necesarias. Realizar los ajustes necesarios para alcanzar los objetivos inicialmente propuestos.

Las características fundamentales del control interno según el Comité de Organizaciones patrocinadoras de la Comisión de Caminos (COSO) (2013) son las siguientes:

- $\quad$ Es un proceso integral que involucra a la dirección y administración, no adyacente a estos.

- Su orientación a los objetivos se considera como un medio, no como un fin en sí mismo.

- Se puede concebir y ejecutar por cada miembro de cualquier nivel de la organización por medio de palabras y/o acciones.

- Su fin primigenio es proporcionar una seguridad razonable del cumplimiento de los objetivos.

Según González (2013), los objetivos del marco se establecen en tres categorías, que permiten a las organizaciones centrarse en diferentes aspectos del control interno, por ello se definen los siguientes objetivos:

- Objetivos operativos: estos objetivos se relacionan con el cumplimiento de la misión y visión de la entidad.

- Objetivos de información: estos objetivos se refieren a la preparación de reportes para uso de la organización y los accionistas, teniendo en cuenta la veracidad, oportunidad y transparencia.

- Objetivos de cumplimiento: están relacionados con el cumplimiento de las leyes y regulaciones a las que está sujeta la entidad. La entidad debe desarrollar sus actividades en función de las leyes y normas específicas.

Los objetivos operativos se relacionan con la eficiencia y efectividad de las operaciones de la organización, los objetivos de información se relacionan con la información financiera y no financiera que puede ser externa o interna, y los objetivos de cumplimiento se relacionan con el cumplimiento de la normatividad, regulación y leyes a las que se encuentra sujeta la organización.

Los componentes del control interno según el COSO (2013) son: 
a. Entorno de Control: Es el conjunto de estructuras, procesos y normas que constituyen la base sobre que desarrollar el control interno de la organización.

b. Evaluación de riesgos: todas las entidades se enfrentan a diferentes riesgos que pueden provenir de fuentes internas o externas. La evaluación de riesgos implica un proceso dinámico e interactivo para evaluar e identificar riesgos con el fin de lograr la consecución de los objetivos. Consiste en como la entidad identifica, analiza y administra el riesgo que afecta el cumplimiento de objetivos.

c. Actividades de control: Son el conjunto de acciones que se establecen a través de procedimientos y políticas que garantizan y contribuyen a que las instrucciones de la dirección se lleven a cabo con el objetivo de mitigar cada uno de los riesgos identificados.

d. Información y comunicación: Se hace necesario la producción de información de calidad y altamente relevante tanto de fuentes externas como internas, para apoyar otros componentes del control interno (p. 6).

Una vez clasificado el control interno, según la Contraloría General de la República (2014) se han desarrollado cada uno de sus componentes:

- El ambiente de control se refiere al "conjunto de normas, procesos y estructuras que sirven de base para llevar a cabo el adecuado Control Interno en la entidad. Los funcionarios, partiendo del más alto nivel de la entidad, deben destacar la importancia del Control Interno, incluidas las normas de conducta que se espera. Un buen ambiente de control tiene un impacto sustantivo en todo el sistema general de Control Interno" (p. 35).

- La evaluación de riesgos comprende los principios de definición de los objetivos e identificación y evaluación de los riesgos, determinación de la gestión de riesgos, evaluación de la probabilidad de fraude y evaluación de sucesos o cambios que afecten al sistema de control interno" (p. 37).

- Las actividades de control se "refieren a aquellas políticas y procedimientos establecidos para disminuir los riesgos que pueden afectar el logro de objetivos de la entidad. Para ser efectivas deben ser apropiadas, funcionar consistentemente de acuerdo a un plan a lo largo de un periodo determinado y tener un costo adecuado, que sea razonable y relacionado directamente con los objetivos del control" (p. 39).

- Las actividades de supervisión del control interno se "refieren al conjunto de actividades de autocontrol incorporadas a los procesos y operaciones de supervisión (o seguimientos) de la entidad con fines de mejora y evaluación" (p. 41).

- La información y comunicación para mejorar el control interno se "refiere a la información necesaria para que la entidad pueda llevar a cabo las responsabilidades de Control Interno que apoyen el logro de sus objetivos. La administración obtiene/genera y utiliza la información relevante y de calidad a partir de fuentes internas y externas para apoyar el funcionamiento de los otros componentes del Control Interno" (p. 40).

- Las actividades de supervisión del Control Interno se "refieren al conjunto de actividades de autocontrol incorporadas a los procesos y operaciones de supervisión (o seguimientos) de la entidad con fines de mejora y evaluación" (p. 41).

El Ministerio de Economía y Finanzas (2010) refiere que:

En lo que respecta a la información financiera, su objetivo es proporcionar información sobre la entidad que informa que sea útil a los inversores, prestamistas y otros acreedores existentes y potenciales para tomar decisiones sobre el suministro de recursos a la entidad. Esas decisiones conllevan comprar, vender o mantener patrimonio e instrumentos de deuda, y proporcionar o liquidar préstamos y otras formas de crédito (p.7).

Asimismo, la Comisión Nacional Supervisora de Empresas y Valores (CONASEV) (2018) manifiesta que se relaciona con "la preparación y presentación de la información financiera, así como su adecuación (...) a las Normas Internacionales de Información Financiera (NIIF) emitidas por el International Accounting Standard Board (IASB) y vigentes en el país" (p. 7).

Por otro lado, la información financiera se encuentra relacionada con los informes financieros en la medida en que los segundos:

Proporcionan información sobre la situación financiera de una entidad que informa, que es información sobre los recursos económicos de la entidad y los derechos de los acreedores contra la entidad que informa. Los informes financieros también proporcionan información sobre los efectos de las transacciones y otros sucesos que cambian los recursos económicos y los derechos de los acreedores de una entidad que informa. Ambos tipos de información proporcionan datos de entrada útiles a la hora de tomar decisiones para proporcionar recursos a una entidad" (Ministerio de Economía y Finanzas, 2010, p. 8). 
La información financiera según Ochoa (2018) es lo siguiente:

Toda información contenida en los estados financieros como el balance general o estado de situación financiera, estado de resultados o de actividades según corresponda, el estado de variaciones en el capital contable o patrimonio contable y el estado de flujo de efectivo que se formulan teniendo como base las normas de información financiera (NIF), así como "información de carácter cualitativo de una entidad económica, proporcionan elementos para realizar el análisis de estados financieros, aplicando los métodos y técnicas para tal efecto; proporcionando información útil sobre los aspectos de liquidez, endeudamiento, rentabilidad, cobertura y actividad, identificando así las fortalezas y debilidades $y$ disponer de elementos para la toma de decisiones (p. 1).

\section{MATERIAL Y MÉTODOS}

En el desarrollo del artículo se ha aplicado el método deductivo, debido a que se parte de datos generales aceptados como válidos para llegar a una conclusión de tipo particular (Hernández, Fernández y Baptista, 2010).

El diseño de la investigación es no experimental-transversal-correlacional. Es no experimental debido a que no se manipularon deliberadamente las variables, así mismo es transversal debido a que se recolectan datos en un solo momento, en un tiempo único. "Su propósito es describir variables y analizar su incidencia e interrelación en un momento dado por lo que es de tipo correlacional" (Hernández, Fernández y Baptista, 2010, p. 90). Es de tipo correlacional porque se evalúa la relación entre dos o más variables, es decir, se intenta explicar cómo se comporta una variable en función de las otras (Vara, 2012).

La población del estudio de investigación fue el personal que labora en el Ministerio de Trabajo y Promoción del Empleo (MTPE), mientras que la muestra consta de 55 personas, tales que: 41 personas laboran en la Unidad Ejecutora 001 Ministerio del Trabajo (MINTRA) - Oficina General De Administración; 4 personas laboran en la Unidad Ejecutora 002 Programa Nacional De Empleo Juvenil Jóvenes Productivos; 5 laboran en Unidad Ejecutora 005 Programa para la Generación de Empleo Social Inclusivo Trabaja Perú; 3 en la Unidad Ejecutora 006 Programa Nacional para la Promoción de Oportunidades Laborales Impulsa Perú; y 2 en la Unidad Ejecutora 007 Programa Para El Mejoramiento Y Ampliación De Los Servicios Del Centro De Empleo Fortalece Perú.

En la investigación, primero se revisó minuciosamente la información contable y financiera, luego se cotejó dicha información con datos históricos lo que permitió establecer una serie de parámetros de entrada para el control interno. Asimismo, se revisaron cada una de las políticas y procedimientos de la entidad en base a los principios del control interno lo que permitió desarrollar un conjunto de recomendaciones en la institución, finalmente se desarrolló la encuesta al personal para determinar el nivel de influencia del control interno en la información financiera en la entidad.

\section{RESULTADOS}

A continuación, se expondrá los resultados de la encuesta realizada apersonas que laboran en las áreas de Contabilidad, Tesorería, Abastecimiento, Almacén, Recursos humanos, Procuraduría Pública, Control patrimonial, Infraestructura, Tecnologías de la información y Comunicación, Fiscalización y Control previo. Así tenemos:

Tabla 1

¿El control interno permite detectar de forma oportuna la ocurrencia de errores dentro de su área de trabajo?

\begin{tabular}{lcc}
\hline $\begin{array}{l}\text { ¿EI control interno permite } \\
\text { detectar de forma oportuna la } \\
\text { ocurrencia de errores dentro } \\
\text { de su área de trabajo? }\end{array}$ & Frecuencia & Porcentaje \\
\hline Sí & 46 & \\
No & 6 & 10,91 \\
No sabe / no opina & 3 & 5,45 \\
Total & 55 & 100,00 \\
\hline
\end{tabular}

Fuente: Elaboración Propia

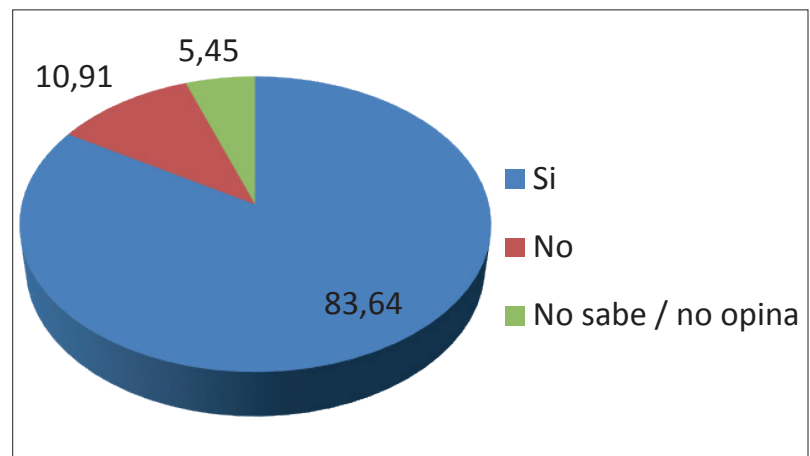

Figura1. Porcentaje de las respuestas a la pregunta ¿El control interno permite detectar de forma oportuna la ocurrencia de errores dentro de su área de trabajo?

Fuente: Elaboración Propia

Según la respuesta de los encuestados, el 83,64\% cree que el control interno permite detectar de forma oportuna la ocurrencia de errores dentro de su área de trabajo y el $10,91 \%$ opina lo contrario y el $5,45 \%$ no sabe no opina. Como se puede apreciar, el resultado nos indica que el 
control interno permite detectar de forma oportuna la ocurrencia de errores para que en el MINTRA los estados financieros sean los adecuados a los fines contables propuestos.

En lo que respecta a la siguiente pregunta, ¿El Órgano de Control institucional realiza evaluaciones periódicas de los controles de los procesos vigentes de la entidad?

Tabla 2

¿El Órgano de Control institucional realiza evaluaciones periódicas de los controles de los procesos vigentes de la entidad?

\begin{tabular}{lcc}
\hline $\begin{array}{l}\text { ¿El Órgano de Control institucio- } \\
\text { nal realiza evaluaciones periódi- } \\
\text { cas de los controles de los proce- }\end{array}$ & Frecuencia & Porcentaje \\
sos vigentes de la entidad? & & \\
\hline $\mathrm{Si}$ & 47 & 85,45 \\
$\mathrm{No}$ & 4 & 7,27 \\
No sabe / no opina & 4 & 7,27 \\
Total & 55 & 100,00 \\
\hline
\end{tabular}

Fuente: Elaboración Propia

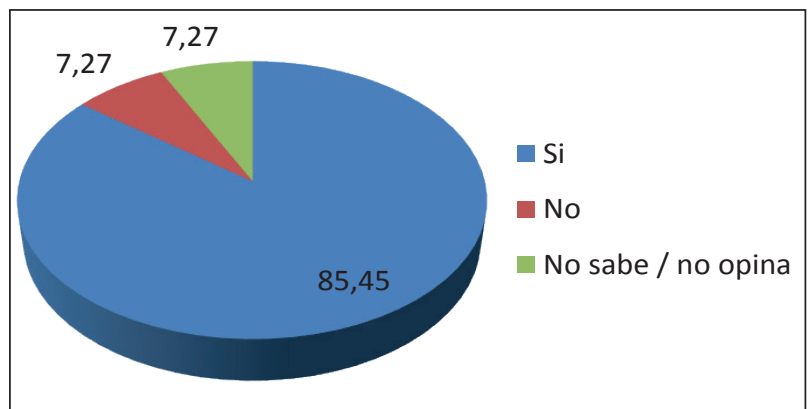

Figura 2. Porcentaje de las respuestas a la pregunta ${ }_{¿} \mathrm{El}$ Órgano de Control institucional realiza evaluaciones periódicas de los controles de los procesos vigentes de la entidad?

Fuente: Elaboración Propia

Según la respuesta de los encuestados, el 85,45\% cree que el Órgano de Control institucional realiza evaluaciones periódicas de los controles de los procesos vigentes de la entidad, el 7,27\% opina lo contrario y el 7,27\% no sabe no opina. Como se puede apreciar, el resultado nos indica que hay opinión que en su mayoría rescata el Órgano de Control institucional realiza evaluaciones periódicas de los controles de los procesos vigentes de la entidad para que asegure que en el MINTRA los estados financieros sean los adecuados a los fines contables propuestos.

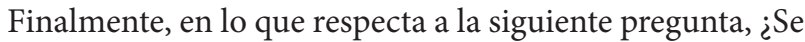
realiza una evaluación periódica de los controles de los procesos vigentes de la entidad por parte del Órgano de Control institucional?
Tabla 3

¿Se realiza una evaluación periódica de los controles de los procesos vigentes de la entidad por parte del Órgano de Control institucional?

\begin{tabular}{|c|c|c|}
\hline $\begin{array}{l}\text { ¿Se realiza una evaluación pe- } \\
\text { riódica de los controles de los } \\
\text { procesos vigentes de la entidad } \\
\text { por parte del Órgano de Control } \\
\text { institucional? }\end{array}$ & Frecuencia & Porcentaje \\
\hline $\mathrm{Si}$ & 35 & 63,64 \\
\hline No & 15 & 27,27 \\
\hline No sabe / no opina & 5 & 9,09 \\
\hline Total & 55 & 100,00 \\
\hline
\end{tabular}

Fuente: Elaboración Propia

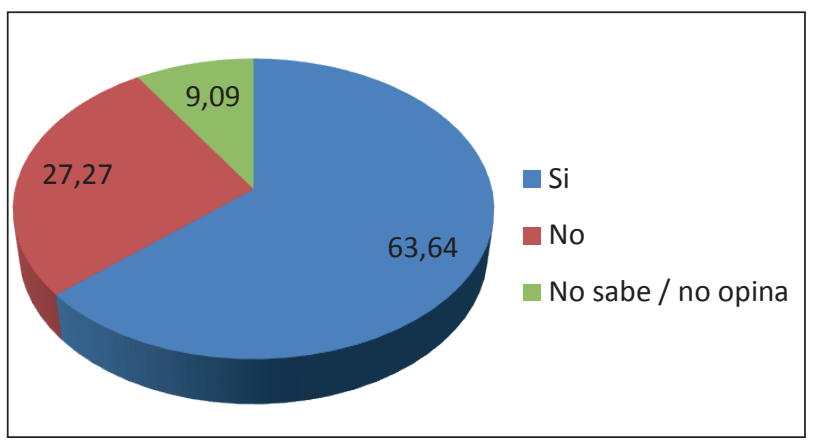

Figura 3. Porcentaje de las respuestas a la pregunta ¿Se realiza una evaluación periódica de los controles de los procesos vigentes de la entidad por parte del Órgano de Control institucional?

Fuente: Elaboración Propia

Según la respuesta de los encuestados, el 63,64\% opina que se realiza una evaluación periódica de los controles de los procesos vigentes de la entidad por parte del Órgano de Control institucional y el 27,27\% opina lo contrario y el 9,09\% no sabe no opina. Como se puede apreciar, el resultado nos indica que se realiza una evaluación periódica de los controles internos de los procesos vigentes de la entidad por parte del Órgano de Control institucional que asegure que en el MINTRA los estados financieros sean los adecuados a los fines contables propuestos.

Finalmente, la hipótesis nula y la hipótesis alternativa se muestran a continuación, para luego proceder a realizar la correlación de las variables, tenemos:

H0: La carencia del control interno bajo los componentes de la Ley 28716 no influye en la fiabilidad de la información financiera del Ministerio de Trabajo y Promoción del Empleo, periodo 2014-2016.

H1: La carencia del control interno bajo los componentes de la Ley 28716 no influye en la fiabilidad de la informa- 
ción financiera del Ministerio de Trabajo y Promoción del Empleo, periodo 2014-2016.

Para realizar la prueba se utilizó el Estadístico: Rho de Spearman, con un nivel de significancia a del 0.05, siendo la regla de decisión la siguiente: a) El nivel de significancia "p" es menor que $\alpha$, se rechaza H0 y b) el nivel de significancia "p" es mayor que $\alpha$, no se rechaza $\mathrm{H} 0$.

Como se puede apreciar en la tabla 4, existe una relación positiva y significativa entre la Carencia del Control Interno y la Fiabilidad de la información financiera del Ministerio de Trabajo y Promoción del Empleo, con un coeficiente de correlación de 0.458 lo cual indica una relación positiva entre las variables. Con el Pvalor obtenido de $0,000 \%$ menor al sig. (5\%) se rechaza la hipótesis nula y queda evidenciado que una carencia de control interno influye en la fiabilidad de la información financiera del Ministerio de Trabajo y Promoción Social.

En lo que respecta a la siguiente hipótesis:

H0: Un adecuado componente de ambiente de control influye favorablemente en los Estados financieros del Ministerio de Trabajo y Promoción del Empleo, periodo 2014-2016.
H1: Un adecuado componente de ambiente de control no influye favorablemente en los Estados financieros del Ministerio de Trabajo y Promoción del Empleo, periodo 2014-2016.

Para realizar la prueba se utilizó el Estadístico: Rho de Spearman, con un nivel de significancia a del 0,05, siendo la regla de decisión la siguiente: a) El nivel de significancia "p" es menor que $\alpha$, se rechaza H0 y b) el nivel de significancia " $p$ " es mayor que $\alpha$, no se rechaza H0. Con el $\mathrm{P}$ valor obtenido de $0,000 \%$ menor al $5 \%$, se rechaza la hipótesis y queda evidenciado que un adecuado ambiente de control influye positivamente en los estados financieros del Ministerio de Trabajo y Promoción Social.

Como se puede apreciar en la tabla 5, existe una relación positiva y significativa entre el componente de ambiente de control y los Estados financieros del Ministerio de Trabajo y Promoción del Empleo, periodo 2014-2016, con un coeficiente de correlación de 0,553 lo cual indica una relación positiva entre las variables.

\section{DISCUSIÓN}

Como se puede apreciar en los resultados de la encuesta al personal que labora en el Ministerio de Trabajo y Pro-

Tabla 4.

Correlación entre la carencia del control interno bajo los componentes de la Ley 28716 y la fiabilidad de la información financiera del Ministerio de Trabajo y Promoción del Empleo

\begin{tabular}{|c|c|c|c|c|}
\hline & & & $\begin{array}{l}\text { Carencia del } \\
\text { Control Interno }\end{array}$ & $\begin{array}{c}\text { Fiabilidad de la información financiera } \\
\text { del Ministerio de Trabajo y Promoción } \\
\text { del Empleo }\end{array}$ \\
\hline \multirow{6}{*}{$\begin{array}{l}\text { Rho de } \\
\text { Spearman }\end{array}$} & \multirow[t]{3}{*}{ Carencia del Control Interno } & Coeficiente de correlación & 1,000 & $458^{* *}$ \\
\hline & & Sig. (bilateral) & 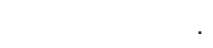 &, 000 \\
\hline & & $\mathrm{N}$ & 55 & 55 \\
\hline & \multirow{3}{*}{$\begin{array}{l}\text { Fiabilidad de la información finan- } \\
\text { ciera del Ministerio de Trabajo y } \\
\text { Promoción del Empleo }\end{array}$} & Coeficiente de correlación &, $458^{* *}$ & 1,000 \\
\hline & & Sig. (bilateral) &, 000 & \\
\hline & & $\mathrm{N}$ & 55 & 55 \\
\hline
\end{tabular}

**. La correlación es significativa en el nivel 0,01 (bilateral).

Tabla 5 .

Correlación entre componente de ambiente de control y los Estados financieros del Ministerio de Trabajo y Promoción del Empleo, periodo 2014-2016

\begin{tabular}{lllrr}
\hline & & \multicolumn{1}{c}{$\begin{array}{c}\text { Componente } \\
\text { de ambiente de } \\
\text { control }\end{array}$} & $\begin{array}{c}\text { Estados financieros del Ministerio } \\
\text { de Trabajo y Promoción del Empleo, } \\
\text { periodo 2014-2016 }\end{array}$ \\
\hline $\begin{array}{l}\text { Rho de } \\
\text { Spearman }\end{array}$ & $\begin{array}{l}\text { Componente de ambiente de } \\
\text { control }\end{array}$ & Coeficiente de correlación & 1,000 &, $553^{* *}$ \\
& & Sig. (bilateral) &, 000 \\
& Estados financieros del Ministe- & Coeficiente de correlación & 55 & 55 \\
& $\begin{array}{l}\text { rio de Trabajo y Promoción del } \\
\text { Empleo, periodo 2014-2016 }\end{array}$ & Sig. (bilateral) &, $553^{* *}$ & 1,000 \\
& $\mathrm{~N}$ &, 000 &. \\
\hline
\end{tabular}

\footnotetext{
**. La correlación es significativa en el nivel 0,01 (bilateral).
} 
moción del Empleo considera que un adecuado sistema de control interno bajo los componentes de ambiente de control mejorará positivamente la consistencia de la información que sustenta el activo, pasivo y patrimonio del Estado de Situación Financiera, la Evaluación de Riesgos mejorará la coherencia y confiabilidad de la información que sustenta los ingresos y gastos el Estado de Gestión, las actividades de control mejorarán positivamente la oportunidad del movimiento patrimonial de la información que sustenta el Estado de Cambios en el Patrimonio Neto, la información y comunicación mejorará positivamente la razonabilidad de la información del movimiento del efectivo que sustenta el Estado de Flujo de Efectivo y el monitoreo mejorará que la información esté libre de errores sustanciales de las notas aclaratorias a los Estados Financieros. En ese sentido, y siguiendo la definición de la Contraloría General de la República (2014), el control interno es un proceso integral efectuado por el titular, funcionario y servidores de una entidad, diseñado para enfrentar los riesgos y para dar seguridad razonable de que, en la consecución de la misión de la entidad, se alcanzaran los objetivos, es decir, es la gestión misma orientada a minimizar los riesgos. Por otro lado, siguiendo a Fonseca (2011), concordamos en lo establecido en el Instituto Americano de Contadores Públicos Certificados (AICPA) en donde el control interno es un proceso efectuado por la alta dirección, las diferentes gerencias y el personal de una entidad, es decir, deben participar todos los miembros de la entidad para brindar seguridad razonable respecto al logro de objetivos.

\section{REFERENCIAS BIBLIOGRÁFICAS}

Comisión Nacional Supervisora de Empresas y Valores (CONASEV) (2018). Manual para la preparación de información financiera. Recuperado de https://www. smv.gob.pe/Uploads/MIF_2008.pdf

Committee of Sponsoring Organizations of the Treadway Commission (COSO) (2013). Control Interno - Marco Integrado. Recuperado de http://doc.contraloria.gob.pe/Control-Interno/Normativa_Asociada/ coso_2013-resumen-ejecutivo.pdf

Contraloría General de la República (2014). Marco conceptual del Control Interno. Recuperado de http://doc. contraloria.gob.pe/Control-Interno/web/documentos/Publicaciones/Marco_Conceptual_Control_Interno_CGR.pdf
Dextre, J., \& Del Pozo, R. (2012). ¿Control de gestión o gestión de control? Contabilidad y Negocios, 7(14), 69-80.

Fonseca, O. (2011). Sistema de Control Interno para organizaciones. Lima: Instituto de Investigación en Accountabililty y Control - IICO.

Gamboa, J., Puente, S., \& Ysidora, P. (2016). Importancia del control interno en el sector público. Revista Publicando, 3(8), 487-503.

González, R. (2013). Marco Integrado de Control Interno. Modelo COSO III Manual del Participante. Recuperado de https://www.ofstlaxcala.gob.mx/doc/material/27.pdf

Gutiérrez, R., \& Católico, D. (2015). E- control en las entidades de la rama ejecutiva del orden nacional en Colombia. Cuadernos Contables, 16(42), 553-578.

Hernández, R., Fernández, C., \& Baptista, P. (2010). Metodología de la Investigación. México D.F.: Mc Graw Hill Education.

Mendoza, W., García, T., Delgado, M., \& Barreiro, I. (2018). El control interno y su influencia en la gestión administrativa del sector público. Dominio de las Ciencias, 4(4), 206-240.

Ministerio de Economía y Finanzas (2010). El Marco Conceptual para la Información Financiera. Lima: Ministerio de Economía y Finanzas. Recuperado de https:// www.mef.gob.pe/contenidos/conta_publ/con_nor_ co/vigentes/nic/ES_GVT_BV2017_conceptual.pdf

Ochoa, M. (2018). Información financiera, base para el análisis de estados financieros. Sonora: Instituto Tecnológico de Sonora. Recuperado de https://www. itson.mx/publicaciones/pacioli/Documents/informacion-financierabase-para-analisis-de-estados-financieros.pdf

Presidencia del Consejo de Ministros (2016). Ley de Control Interno de las entidades del Estado LEY $N^{\circ}$ 28716. Lima: Presidencia del Consejo de Ministros Recuperado de http://www.pcm.gob.pe/wp-content/ uploads/2016/06/Ley_N_28716.pdf

Vara, A. (2012). Desde la idea hasta la sustentación: Siete pasos para una tesis exitosa. Un método efectivo para las ciencias empresariales (3ra. ed.). Lima: Instituto de Investigación de la Facultad de Ciencias Administrativas y Recursos Humanos. 